\title{
Three-Dimensional Numerical Simulation of Stably Stratified Flows over a Two-Dimensional Hill
}

\author{
Takanori Uchida \\ Research Institute for Applied Mechanics, Kyushu University, Kasuga-shi, Japan \\ Email: takanori@riam.kyushu-u.ac.jp
}

How to cite this paper: Uchida, T. (2017) Three-Dimensional Numerical Simulation of Stably Stratified Flows over a Two-Dimensional Hill. Open Journal of Fluid Dynamics, 7, 579-595.

https://doi.org/10.4236/ojfd.2017.74039

Received: October 28, 2017

Accepted: December 19, 2017

Published: December 22, 2017

Copyright $\odot 2017$ by author and Scientific Research Publishing Inc. This work is licensed under the Creative Commons Attribution International License (CC BY 4.0).

http://creativecommons.org/licenses/by/4.0/

\begin{abstract}
Stably stratified flows over a two-dimensional hill are investigated in a channel of finite depth using a three-dimensional direct numerical simulation (DNS). The present study follows onto our previous two-dimensional DNS studies of stably stratified flows over a hill in a channel of finite depth and provides a more realistic simulation of atmospheric flows than our previous studies. A hill with a constant cross-section in the spanwise $(y)$ direction is placed in a $3-D$ computational domain. As in the previous $2-D$ simulations, to avoid the effect of the ground boundary layer that develops upstream of the hill, no-slip conditions are imposed only on the hill surface and the surface downstream of the hill; slip conditions are imposed on the surface upstream of the hill. The simulated 3-D flows are discussed by comparing them to the simulated 2- $D$ flows with a focus on the effect of the stable stratification on the non-periodic separation and reattachment of the flow behind the hill. In neutral ( $K=0$, where $K$ is a non-dimensional stability parameter) and weakly stable $(K=0.8)$ conditions, $3-D$ flows over a hill differ clearly from 2- $D$ flows over a hill mainly because of the three-dimensionality of the flow, that is the development of a spanwise flow component in the $3-D$ flows. In highly stable conditions $(K=1,1.3)$, long-wavelength lee waves develop downstream of the hill in both 2- $D$ and 3- $D$ flows, and the behaviors of the 2- $D$ and 3- $D$ flows are similar in the vicinity of the hill. In other words, the spanwise component of the 3-D flows is strongly suppressed in highly stable conditions, and the flow in the vicinity of the hill becomes approximately two-dimensional in the $x$ and $z$ directions.
\end{abstract}

\section{Keywords}

Finite-Difference Method, Stably Stratified Flows, Two-Dimensional Hill 


\section{Introduction}

The atmospheric boundary layer is often characterized by vertical variations of air density. In density stratified layers such as the surface inversion layer that is frequently observed at nighttime, the air density decreases with height. In stably stratified conditions, negative buoyancy forces are present. As a result, when air flows over simple or complex topographies in stably stratified conditions, waves and other fluid phenomena emerge that are not observed in neutrally stratified conditions [1]-[14]. Internal gravity waves are one such phenomenon [1]. When an atmospheric layer (lid) that can reflect internal gravity waves forms at the top of the surface inversion layer, the energy of internal gravity waves (lee waves) excited by the topography is trapped between the ground surface and the atmospheric layer (lid). The trapped internal gravity waves have a significant influence on the flow over the topography. Understanding these wave and other fluid phenomena is necessary to predict wind characteristics in the vicinity of both simple and complex topographies for numerous practical applications. These applications include effective use of wind energy, prediction of air pollutant advection and dispersion, and issues related to high wind speeds such as topography-induced wind disasters (disasters due to high local wind) [2].

In the past, the authors have conducted numerical studies on stably stratified flows over a two-dimensional hill with a model based on the finite differencing method [3]-[11]. In these studies, in order to simulate flow over a hill with an upper atmospheric lid aloft, the hill was set in a channel of finite depth. Uchida and Ohya [10] clarified the relationship between the unsteady flow that emerges over the hill and the behavior of columnar disturbances that propagate upstream of the hill. The same work showed that the characteristics of unsteady flow over a hill were highly influenced by variables related to the non-linearity of the flow such as the Reynolds number, the hill height, the channel depth, the blockage ratio, and the topography configuration parameters. Uchida and Ohya [10] also elucidated the effect of stable stratification on the non-periodic separation and reattachment of the flow behind the hill. Uchida and Ohya [8] investigated the influence of the streamwise grid resolution on computational results and proposed an effective numerical computational method for analyses of flow over a hill in a stably stratified fluid.

Motivated by the issues of topography-induced wind disasters in high wind speed conditions, Uchida and Ohya [7] [9] simulated flows over a hill with uniform inflow speed and evaluated the percentage increase of the wind velocity in areas on the hill with locally increased wind speed. In highly stable conditions, breaking lee waves emerged in the vicinity of the hill. A region of extremely high wind velocity formed downstream of the breaking lee waves, and the wind velocity in the region exceeded the inflow velocity [9]. The series of studies introduced above was conducted with a $2-D$ direct numerical simulation (DNS), and some of the effects of stable stratification on the spanwise flow structure (three-dimensionality of the flow along the spanwise component) remain unknown. 
The present study investigates the characteristics of 3- $D$ flows over a hill, which are more realistic than the $2-D$ flows studied previously. The effect of the ground boundary layer that develops over the upstream side of the hill is not considered as in our previous studies [5], that is, slip conditions are imposed on the surface upstream of the hill, and no-slip conditions are imposed only on the surface of the hill and the surface downstream of the hill. With this set of boundary conditions, numerical simulations are conducted with DNS for a Reynolds number of 2000 as in our previous studies [5]. The simulated 3- $D$ flows will be discussed in terms of the differences and similarities with the simulated 2-D flows, particularly in terms of the effect of the stable stratification on the non-periodic separation and reattachment of the flow behind the hill.

\section{Governing Equations for Stratified Flows over a Hill and Numerical Computational Method}

Three-dimensional numerical simulations are performed for incompressible density stratified flows (stably stratified flows) over a 2-D hill with a constant cross-section in the spanwise $(y)$ direction. The computational domain and the coordinate system used for the simulations are shown in Figure 1. The dimensions of the computational domain are $100 h \times 8 h \times 6 h$ in the streamwise $(x)$, spanwise $(y)$ and vertical $(z)$ directions, respectively, where $h$ is the height of the hill. To simulate the flow over a hill with an atmospheric lid aloft, the hill is set within a channel of finite depth, $H$. The ratio of the hill height to the channel depth, $h / H$, is set to 6 in the present study. Because columnar disturbances develop at the hill and propagate upstream, the hill is placed far downstream from the inflow boundary, i.e. $60 \mathrm{~h}$, so that the arrival of the disturbances at the inflow boundary is delayed [10]. The cross-section of the hill is defined by

$$
z(x)=h \times\{1+\cos (\pi x / a)\} / 2
$$

for $|x| \leq a$, where the parameter a specifies the steepness of the hill. In the present study, the parameter $a$ is set to $a=h(=1)$ to model a steep $2-D$ hill. The density and pressure fields are decomposed into base and perturbation components. Far upstream of the hill, the base density field $\rho_{B}$ is defined so that the density decreases linearly in the vertical direction $(z)$ as $\mathrm{d} \rho_{B} / \mathrm{d} z=-1$. The base pressure field that is required for hydrostatic equilibrium with the base density field is referred to as $p_{B}$. A vertically uniform profile of wind velocity, $U$, is imposed on the flow approaching the hill. Furthermore, the Boussinesq approximation is applied so that the density is assumed to be constant except in the

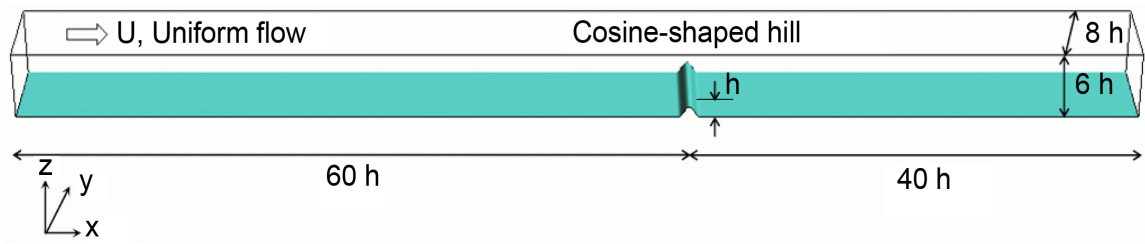

Figure 1. Computational domain and coordinate system. 
buoyancy term.

The five unknown variables to be solved for are the velocity components, $u_{i}$ $(=u, v, w)$, the deviation of pressure from the base pressure field, $p^{\prime}\left(=p-p_{B}\right)$, and the deviation of the density from the base density field, $p^{\prime}\left(=\rho-\rho_{B}\right)$. The governing equations for density stratified flows over a hill consist of the continuity equation, the Navier-Stokes equation, and the density equation. The non-dimensionalized forms of these governing equations can be expressed as

$$
\begin{gathered}
\frac{\partial u_{i}}{\partial x_{i}}=0 \\
\frac{\partial u_{i}}{\partial t}+u_{j} \frac{\partial u_{i}}{\partial x_{j}}=-\frac{\partial p}{\partial x_{i}}+\frac{1}{R e} \frac{\partial^{2} u_{i}}{\partial x_{j} \partial x_{j}}-\frac{\rho}{F r^{2}} \delta_{i 3} \\
\frac{\partial \rho}{\partial t}+u_{j} \frac{\partial \rho}{\partial x_{j}}=w
\end{gathered}
$$

where the prime notations ( ) indicating the deviation of the density and pressure from their base values have been omitted, and the subscripts represent Einstein notation. In Equation (3), the non-dimensional numbers $R e$ and $F r$ indicate the Reynolds number $\left(=\rho_{0} U h / \mu\right)$ and the Froude number $(=U / N h)$, respectively, where $\rho_{0}$ is the reference density, $\mu$ is the coefficient of viscosity, $N$ is the buoyancy frequency defined as $N^{2}=-\left(g / \rho_{0}\right)\left(\mathrm{d} \rho_{B} / \mathrm{d} z\right)$, and $g$ is the acceleration of gravity. Because a hill of height $h$ is set within a channel of finite depth, $H$, in the present study, the non-dimensional stability index $K(=N H / \pi U)$ is adopted to express the stability of the flow. $F r$ and $K$ are related by $F r: K=H / \pi h$, thus, the smaller the value of $F r$ or the larger the value of $K$, the more stable the stratification of the flow.

In order to avoid numerical instability and to achieve highly accurate predictions of the flow over the hill in stably stratified conditions, the DNS simulations are performed using collocated grids in a general curvilinear coordinate system $(\xi-\eta-\zeta)$. In the collocated grid system, staggered allocation is used: velocity components, $u_{p}$ pressure, $p$, and density, $\rho$, are defined at the cell center, and variables that result from the contravariant velocity components, $U_{i}(=U, V, W)$, multiplied by the Jacobian, $J$, are defined at the cell faces. As for the computational technique of the DNS simulations, the finite-difference method (FDM) is adopted. The pressure-velocity coupling algorithm and the time marching method are based on the fractional-step method [15] and the first-order explicit Euler method, respectively. The Poisson's equation for pressure is solved by the successive over-relaxation (SOR) method. For discretization of all the spatial terms except for the convective terms in Equations (3) and (4), a second-order central difference scheme is applied. The convective terms are discretized with the use of the third-order upwind differencing scheme proposed by Kawamura and Kuwahara (the Kawamura-Kuwahara scheme) [16]. For the fourth-order central differencing term that appears in the discretized convective terms, the interpolation method of Kajishima is applied (four-point differencing and 
four-point interpolation) [17].

The number of computational grid points in the $x, y$, and $z$ directions is $221 \times$ $81 \times 101$, respectively. The cells in the vicinity of the hill are the smallest, and their dimensions are $\Delta x_{\min }=0.04 h$ and $\Delta z_{\min }=0.003 h$. The spanwise $(y)$ dimension of the computational domain is divided equally into $\Delta y=0.1 \mathrm{~h}$. A number of grid resolutions were tested, and it was confirmed that the results of the present study are nearly independent of the choice of grid resolution. Figure 2 shows the streamwise cross-section of the hill at $y=0$ and the computational grid in the vicinity of the hill. As for the initial conditions, an impulsive start ( $u=$ $U=1, v=w=p=\rho=0$ ) is applied. For the boundaries, the following conditions are imposed: slip conditions for the upper and side boundaries and the surface upstream of the hill; non-slip conditions on the hill surface and the surface downstream of the hill; uniform inflow conditions for $U$ across the inflow boundary; and convective outflow conditions for the outflow boundary. Slip conditions are applied for the surface upstream of the hill to avoid the effect of the ground boundary layer that develops upstream of the hill in order to clarify the effect of the topography on the flow. As in our previous research [3]-[10], simulations are performed at a Reynolds number $\left(=\rho_{0} U h / \mu\right)$ of 2000 , where $h$ and $U$ represent the hill height and the uniform inflow velocity, respectively. The non-dimensional time step used in the model is $\Delta t=0.002$.

Four cases are considered for the simulations: 1 ) neutral stratification at $K=0$ $(F r=\infty), 2)$ weakly stable stratification at $K=0.8(F r \approx 2.39), 3)$ highly stable stratification at $K=1(F r \approx 1.91)$, and 4$)$ highly stable stratification at $K=1.3$ ( $F r \approx$ 1.47). The readers are advised to refer to Uchida and Ohya [10] for a discussion of the ways in which the simulated flow is influenced by variables and parameters related to the non-linearity of the flow such as the Reynolds number, the blockage ratio, and the topography configuration parameters.

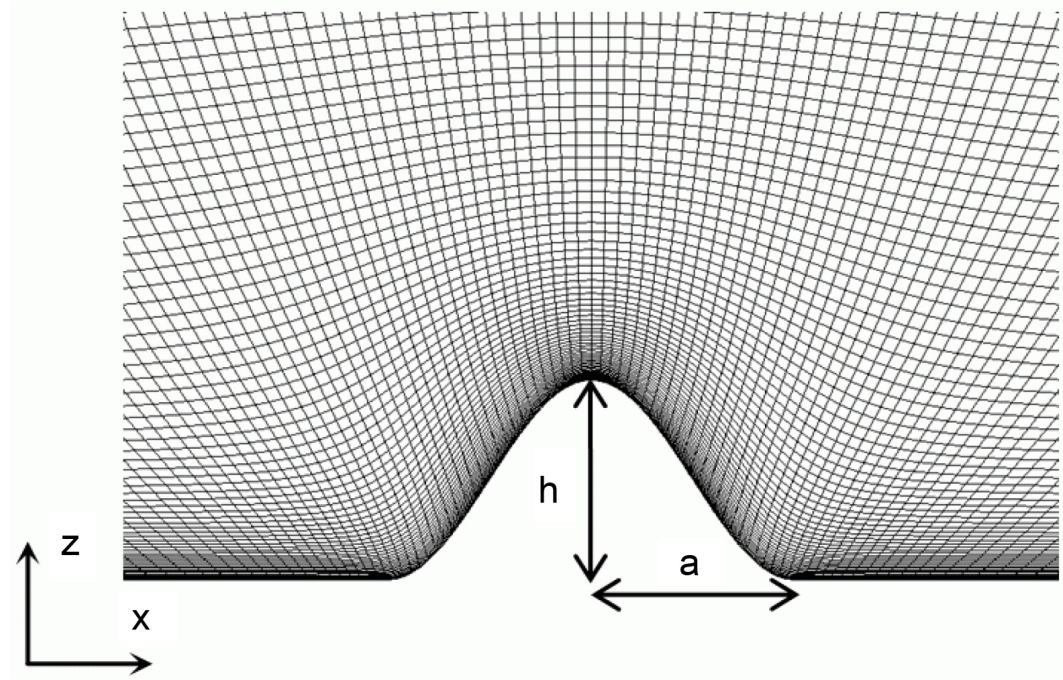

Figure 2. The $x-Z$ cross-section of the computational grid in the vicinity of the hill at $y=0$. 


\section{Simulation Results and Discussions}

\subsection{Temporal Change of the Drag Coefficient, $C d$, of the Hill and the Change of $C d$ with Respect to the Stability, $K(0 \leq K \leq 1.3)$}

The 3- $D$ simulation results from the streamwise cross-section at $y=0$ are discussed by comparing them to $2-D$ simulation $(221 \times 101$ grid points) results. First, the temporal change of the drag coefficient, $C d$, of the hill is compared between the 2- $D$ and 3- $D$ flows. The comparisons are made for the four cases of stability, $K$. The drag coefficient of the streamwise cross-section $(y=0)$ of the hill is evaluated from

$$
C d=2 \int_{S} p z_{\xi} \mathrm{d} \xi-\frac{2}{R e} \int_{S} \omega_{y} x_{\xi} \mathrm{d} \xi
$$

where $p$ is the pressure, $\omega_{y}$ is the spanwise $(y)$ component of the vorticity $(=$ $\partial u / \partial z-\partial w / \partial x)$, and $x_{\xi}$ and $z_{\xi}$ are metrics. Integration is performed over the surface of the hill $|x| \leq a$.

In both neutral $(K=0)$ and weakly stable $(K=0.8)$ conditions, the value of $C d$ in the 2- $D$ simulations fluctuates periodically in a continuous manner (Figure 3(a) \& Figure 3(b)). These fluctuations are associated with the periodic and persistent shedding of large vortices downstream from the recirculation region (the separation bubble) formed behind the hill [10]. The vortex-shedding period for $K=0.8$ is slightly longer than that for $K=0$. A periodic deformation is observed within the cycle of the $C d$ fluctuation due to the secondary vortices induced within the separation bubble behind the hill [10]. In contrast, the behavior of $C d$ from the 3-D simulations in both stability cases changes with time and can be classified into 3 regimes [18]. For $t \leq 20$, the fluctuation pattern of $C d$ is similar to that for the $2-D$ simulations. For $20<t \leq 60$, the amplitude of the $C d$ fluctuation as well as the value of $C d$ decreases rapidly with time. The flow in this time period is in a transitory state, in which three dimensional flow develops with the emergence of the spanwise $(y)$ component of the flow. For $t>60$, the 3- $D$ flow has fully developed, and the behavior of the $C d$ fluctuation differs noticeably from that in the initial period: both the amplitude of the $C d$ variation and the value of $C d$ are quite small although periodic fluctuations of $C d$ remain. As a result, the time-averaged value of $C d$ of the fully developed 3- $D$ flow is significantly lower than that of the 2- $D$ flow as discussed later (Figure 4).

In highly stable conditions $(K=1,1.3)$, the trends of the fluctuating $C d$ in the initial period are similar in the 2- $D$ and 3-D simulations (Figure 3(c) \& Figure 3(d)). Even after 3- $D$ flows have developed with the emergence of the spanwise (y) component of the flow, the behaviors of $C d$ remain qualitatively similar between the 2- $D$ and 3- $D$ simulations. This finding suggests that the spanwise $(y)$ component of the 3- $D$ flow is suppressed in the vicinity of the hill with increasing values of $K$, which results in the quasi-two-dimensional flow behavior in the $x$ and $z$ directions around the hill (Section 3.4). Furthermore, in the case of $K=$ 1 , the periodic fluctuation of $C d$ diminishes rapidly with time, and the value of 


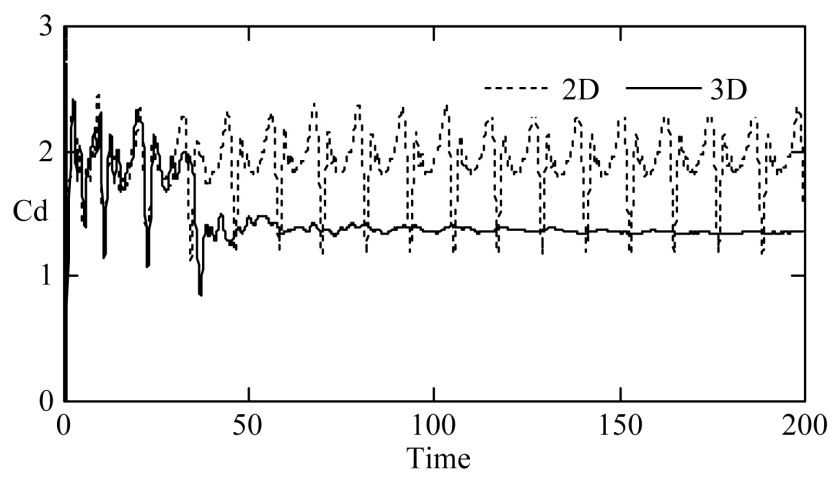

(a) $\mathrm{K}=0$

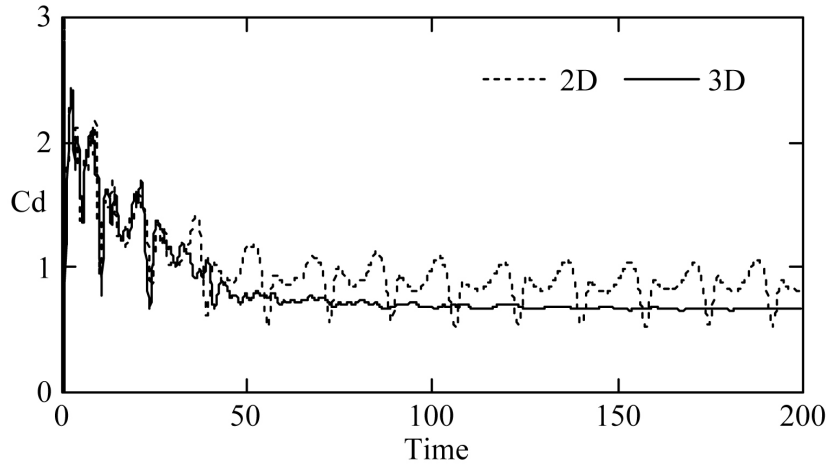

(b) $\mathrm{K}=0.8$

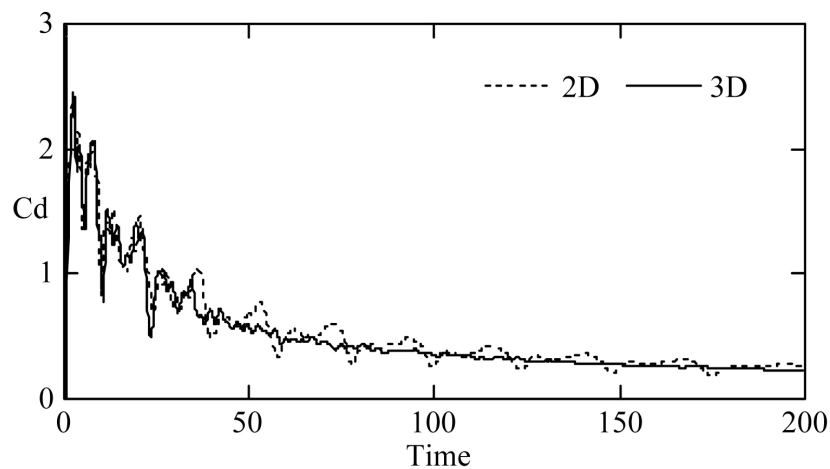

(c) $\mathrm{K}=1$

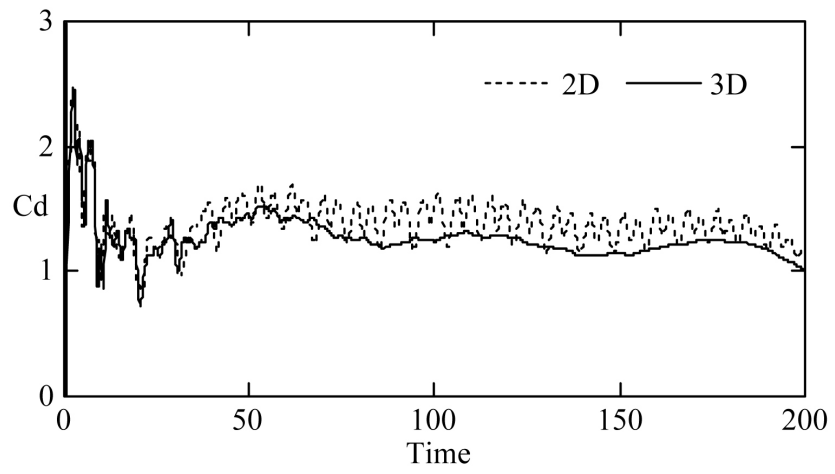

(d) $\mathrm{K}=1.3$

Figure 3. Temporal change of the drag coefficient, $C d$, evaluated for the hill surface $(|x| \leq a), R e=2000$. The horizontal axis indicates the non-dimensional time $(=t /(h / U))$. 


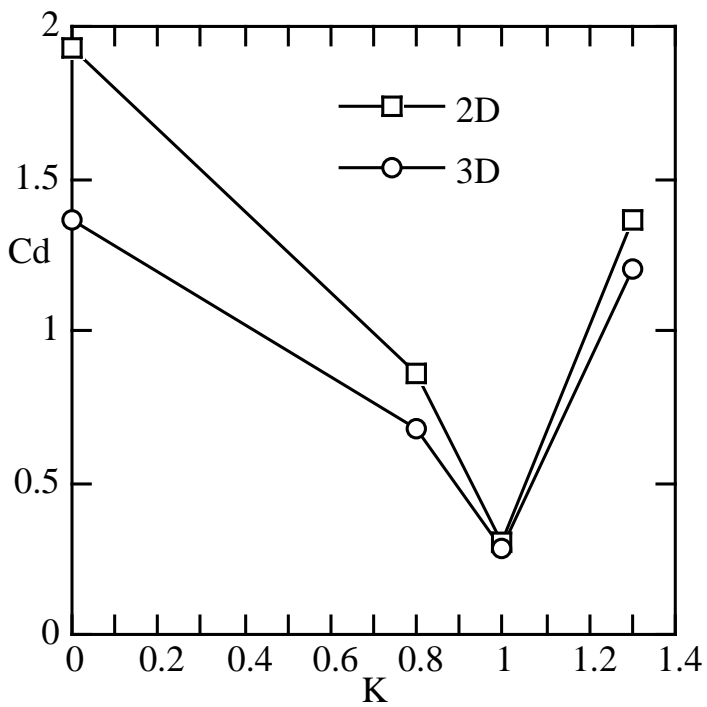

Figure 4. Relationship between the stratification parameter, $K$, and the time-averaged value (non-dimensional time $=100-200)$ of the drag coefficient, $C d, R e=2000$.

$C d$ subsequently becomes approximately constant both in the 2- $D$ and 3- $D$ simulations (Figure 3(c)). This temporal change in the behavior of $C d$ is caused by the development of long-wavelength lee waves that are excited downstream of the hill. As the lee waves develop, the periodic shedding of large vortices from the separation bubble behind the hill becomes suppressed [10], and the flow over the hill reaches an approximately steady state. In the case of $K=1.3$, a long-period oscillation of $C d$ is observed in both the 2-D and 3-D simulations (Figure 3(d)). This long-period oscillation of $C d$ occurs as columnar disturbances are shed from the hill upstream almost periodically [10]. Because these shed disturbances periodically modify the approach flow immediately upstream of the hill, the period of the wave-disturbance propagation upstream of the hill (shedding period) and that of the oscillation of $C d$ at the hill surface are approximately synchronized [10]. The structure of the columnar disturbance upstream of the hill in the 3- $D$ simulation is uniform in the spanwise $(y)$ direction. In the 2-D simulation, long-period oscillations of $C d$ are superimposed on short-period fluctuations of $C d$, suggesting the re-emergence of vortex shedding from the separation bubble behind the hill [10].

Finally, the relationship between the stability parameter $K$ and the time-averaged values of $C d$ is examined for the 2-D and 3-D simulations (Figure 4). The time-averaged values are computed for the non-dimensional time period $t=100$ 200. As discussed earlier, the time-averaged values of $C d$ differ noticeably between the 2-D and 3- $D$ simulations for $K=0$ and 0.8. However, the values are similar in the simulations for $K=1$ and 1.3. Consistent with the results of Uchida and Ohya [10], both in the 2- $D$ and 3- $D$ simulations, the time-averaged value of $C d$ decreases as $K$ increases for low values of $K$, reaches a minimum value at the integer value of $K=1$ and increases as $K$ increases to $K=1.3$. 


\subsection{Flow over the Hill ( $0 \leq K \leq 1.3)$, Instantaneous Field}

The characteristics of the instantaneous flow field from the 2- $D$ and 3- $D$ simulations are investigated. Figure 5 illustrates the streamlines of released virtual fluid particles, and Figure 6 shows the spanwise $(y)$ vorticity, $\omega_{y}$ of the flow fields in Figure 5. In neutral $(K=0)$ and weakly stable $(K=0.8) 2-D$ flows, as discussed earlier, large 2- $D$ eddies (indicated by arrows in Figure 5(a) \& Figure 5(b)) are shed from the separation bubble formed behind the hill and are advected downstream [10]. No significant differences can be seen between the cases of $K=0.8$ and $K=0$ in terms of the size of the separation bubble formed behind the hill and the vorticity distribution within the bubble. However, the circulation of the large eddies downstream away from the hill in the 2- $D$ flow is weakened (reduced number of isolines) at $K=0.8$ compared to that at $K=0$ (Figure 6(a) \& Figure $6(\mathrm{~b}))$. Therefore, at $K=0.8$, the effect of stable stratification on the flow is not obvious immediately downstream of the hill and becomes apparent further downstream from the hill.

The characteristics of the 3-D flows at $K=0$ and 0.8 differ noticeably from those of the 2- $D$ flows [18]. Although vortices are also shed periodically from the hill in the 3- $D$ flows, the behaviors of the shed vortices differ significantly from

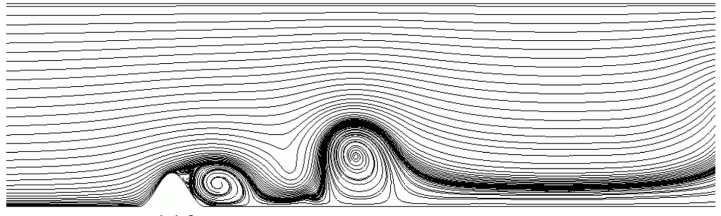

$\mathrm{t}=110$

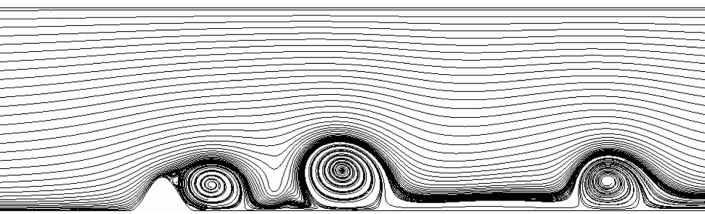

$\mathrm{t}=112$

$\uparrow$

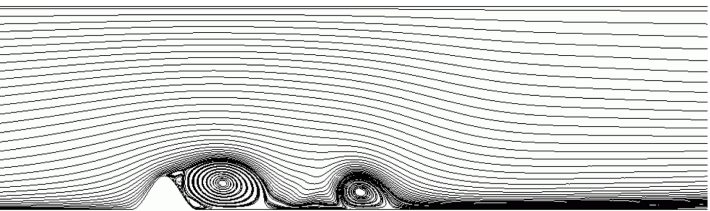

$\mathrm{t}=110$

(c) $\mathrm{K}=1$

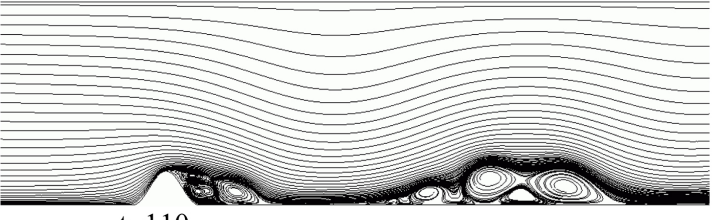

$\mathrm{t}=110$

(d) $\mathrm{K}=1.3$

(b) $\mathrm{K}=0.8$

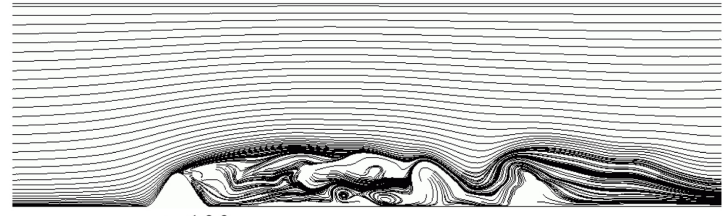

$\mathrm{t}=100$

a) $\mathrm{K}=0$

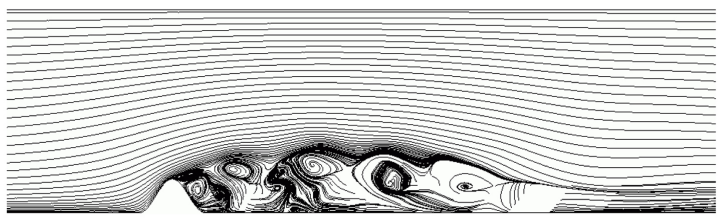

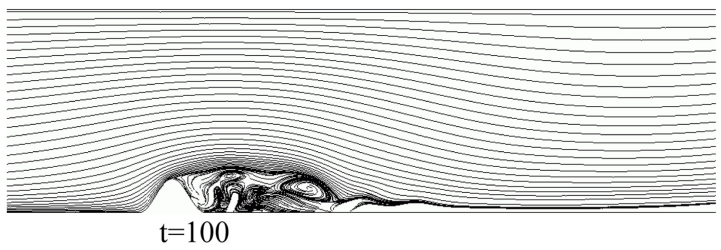

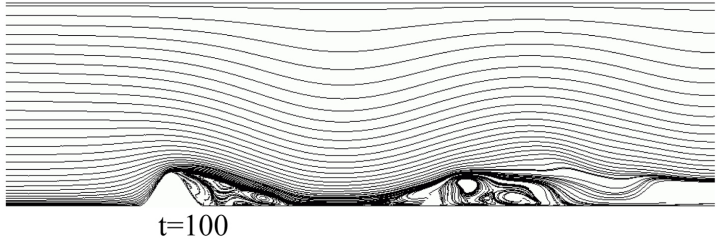

$\mathrm{t}=100$

Figure 5. Streamlines of the instantaneous flow field (left: $2-D$ simulations; right: $3-D$ simulations), $R e=2000$. For the 3-D simulations, streamlines from the $x$ - $Z$ cross-section at $y=0$ are shown. 


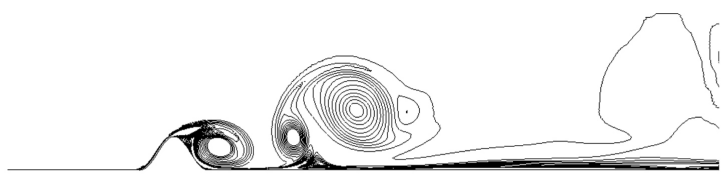

$\mathrm{t}=110$

(a) $\mathrm{K}=0$

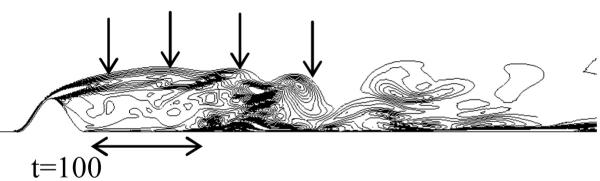

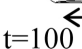

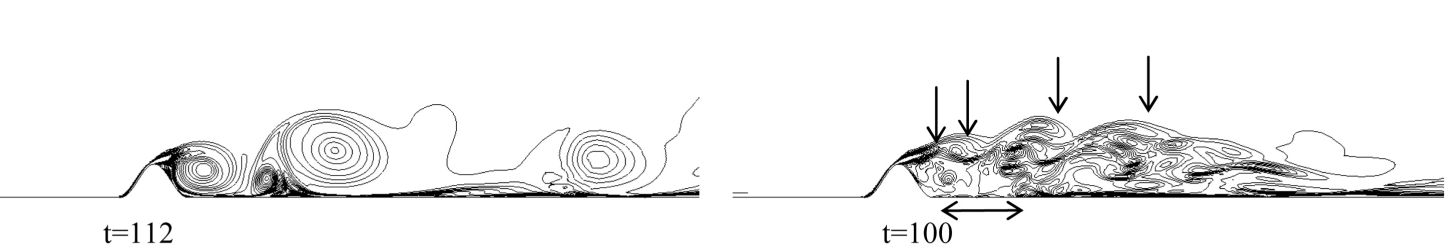

(b) $\mathrm{K}=0.8$

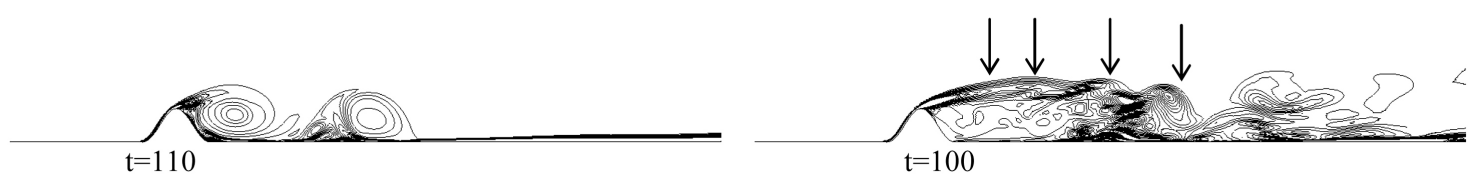

(c) $\mathrm{K}=1$

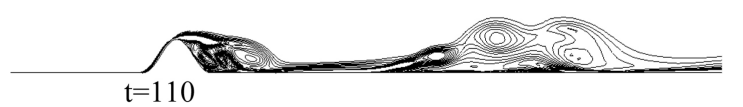

(d) $\mathrm{K}=1.3$

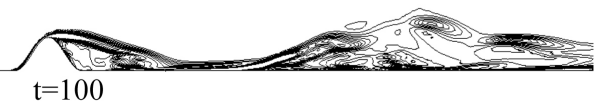
$\mathrm{t}=100$

Figure 6. Contour plots of the spanwise $(y)$ component of vorticity, $\omega_{y}$, for the instantaneous flow field of Figure 5. (left: 2- $D$ simulations; right: 3- $D$ simulations). Twenty equally-spaced isolines are shown between $\omega_{y}=-5$ and $\omega_{y}=$ $5, R e=2000$. For the 3- $D$ simulations, contour plots from the $x$ - $Z$ cross-section at $y=0$ are shown.

those of the 2- $D$ flows. Specifically, in the 3- $D$ flow, the shear layers which separate from the vicinity of the top of the hill, do not reattach on the downstream slope of the hill, and also curl upward some distance downstream of the hill. Subsequently, vortices form that are not connected to the surface downstream of the hill (indicated by arrows in Figure 6(a) \& Figure 6(b)). These vortices are then shed and advected downstream. At $K=0.8$, due to the effect of stratification, obvious vortex shedding is suppressed downstream of the area immediately behind the hill. In both the cases of $K=0$ and 0.8 , the vorticity of the flow is nearly constant in the area immediately behind the hill (horizontal arrows in Figure 6(a) \& Figure 6(b)); that is, a region of dead air forms behind the hill, in which little flow change is observed. The presence of this region leads to the small magnitude of the $C d$ fluctuations as shown in Figure 3(a) \& Figure 3(b).

In the cases of $K=1$ and 1.3, that is, in highly stable conditions, lee waves are excited downstream of the hill in both the 2- $D$ and 3-D simulations as suggested by the streamlines (Figure 5(c) \& Figure 5(d)). The wavelength of the lee waves decreases with increasing stability. This finding together with the temporal change in the drag coefficient, $C d$, of the hill surface (Figure 3 ) reveals the fol- 
lowing: at $K=1$, the periodic vortex shedding from the separation bubble formed behind the hill becomes suppressed with the development of long-wavelength lee waves in both the 2- $D$ and 3- $D$ simulations. In the 3-D simulation at $K=1$, the curl of the separated shear layers and detached eddies formed by the separated shear layers (see arrows in Figure 6(c)) are clearly observed as in the 3-D simulations at $K=0$ and 0.8 . Although the eddies are not connected to the surface downstream of the hill, they are not shed downstream from the hill due to the strong downward flow of the lee-waves. In the 2- $D$ flow at $K=1.3$, vortex shedding re-emerges, however, the shed vortices are rapidly damped by the effect of the stable stratification and vanish. In contrast, at the same stability, no vortex shedding is observed in the 3-D simulation. Furthermore, at $K=1.3$ in both the 2- $D$ and 3- $D$ simulations, separated flow (rotors) is induced above the ground downstream of the hill as a result of the upward flow of the lee waves. Overall, the qualitative behaviors of the 2- $D$ and 3- $D$ flows over the hill are quite similar both at $K=1$ and 1.3. This trend is more obvious at $K=1.3$ as suggested by later discussions (Figures 7-9). Further details will be addressed in conjunction with Figure 10 and Figure 11.

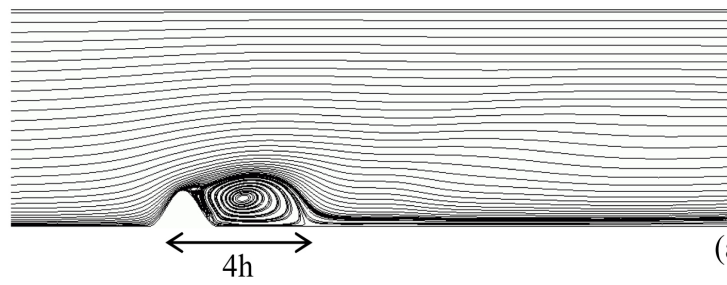

(a) $\mathrm{K}=0$
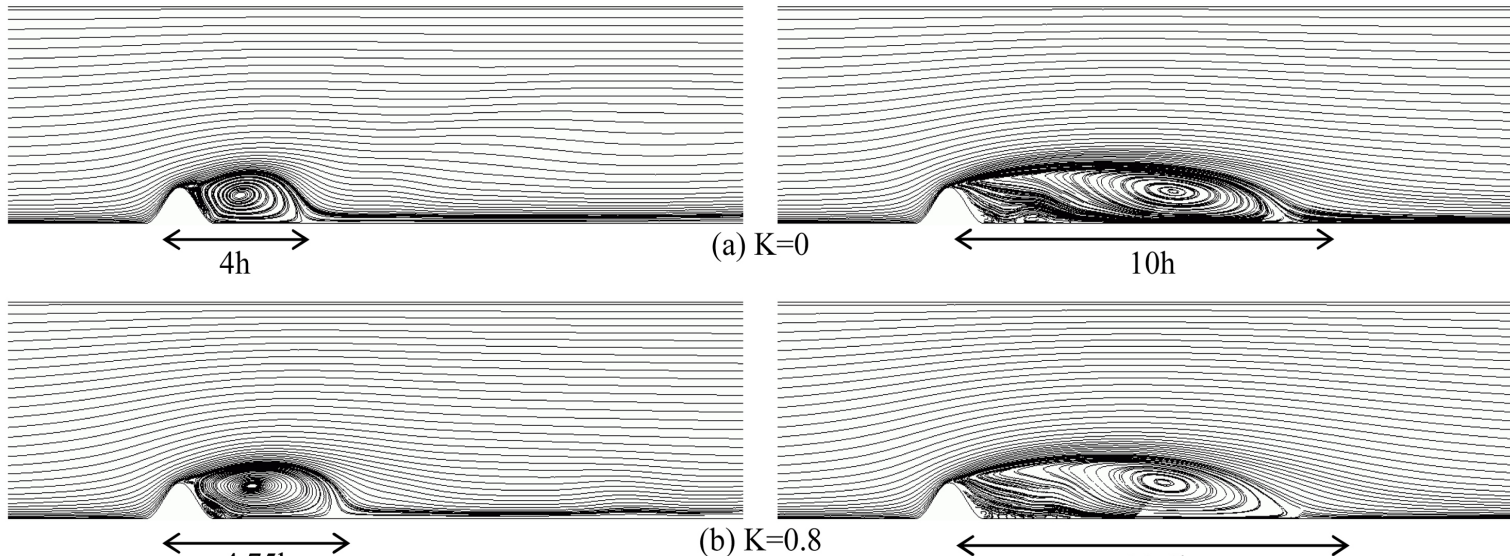

(b) $\mathrm{K}=0.8$
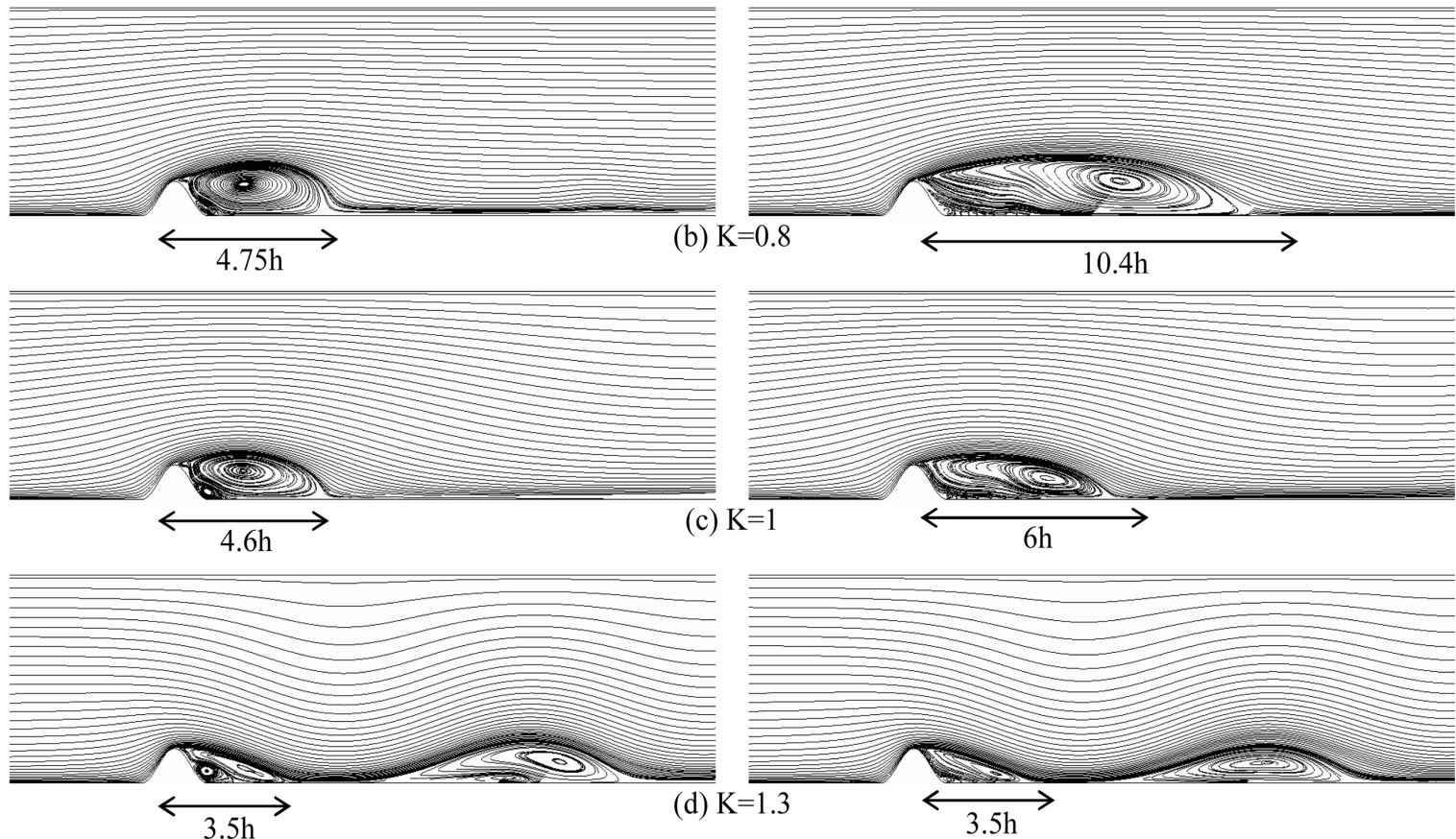

Figure 7. Streamlines of the flow averaged over the time period between the non-dimensional time $=100-200$. (left: 2- $D$ simulations; right: 3-D simulations), $R e=2000 . h$ indicates the hill height. In the 3-D simulations, spanwise ( $y)$ spatial averaging has also been performed. 
(a) $\mathrm{K}=0$
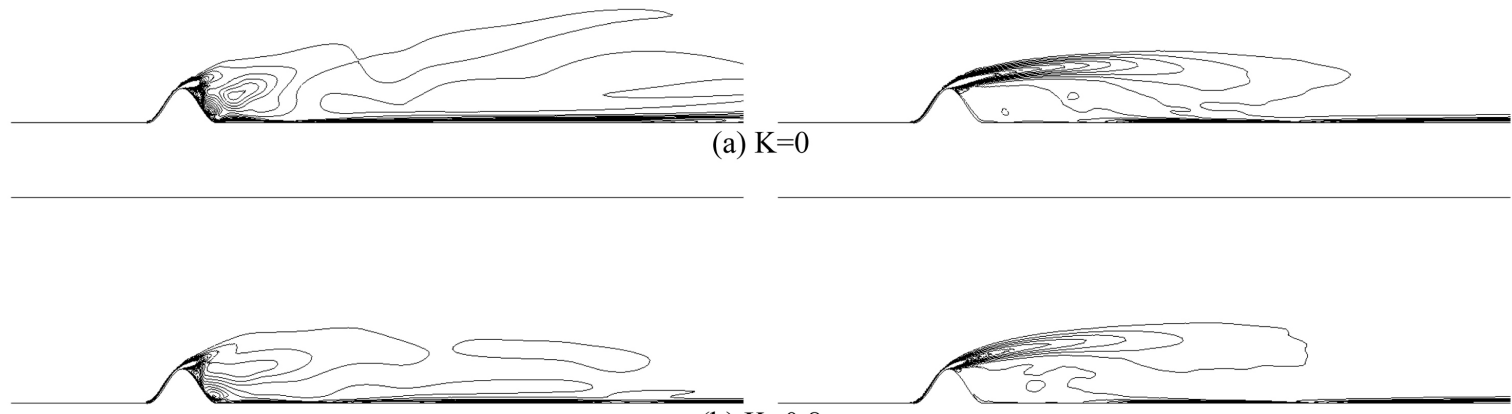

(b) $\mathrm{K}=0.8$

(c) $\mathrm{K}=1$

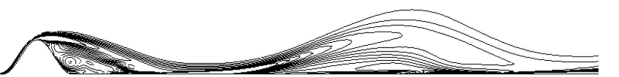

(d) $\mathrm{K}=1.3$

Figure 8. Contour plots of the spanwise $(y)$ component of vorticity, $\omega_{y}$, for the time-averaged flow field of Figure 7. (left: 2- $D$ simulations; right: $3-D$ simulations). Twenty equally-spaced isolines are shown between $\omega_{y}=-5$ and $\omega_{y}=5$, $R e=2000$.

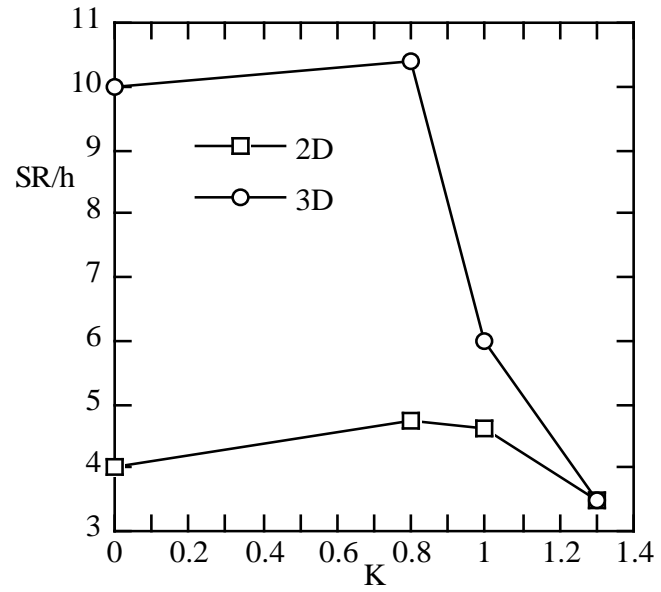

Figure 9. Relationship between the stability parameter, $K$, and the non-dimensional downstream distance, $S R / h$. $S R$ represents the downstream distance from the hill center $(x=0)$ at which the separated shear layer is reattached, and $h$ represents the hill height. The value of $S R / h$ is evaluated from the time-averaged (non-dimensional time $=100-200$ ) flow field of Figure 7, $R e=2000$. 

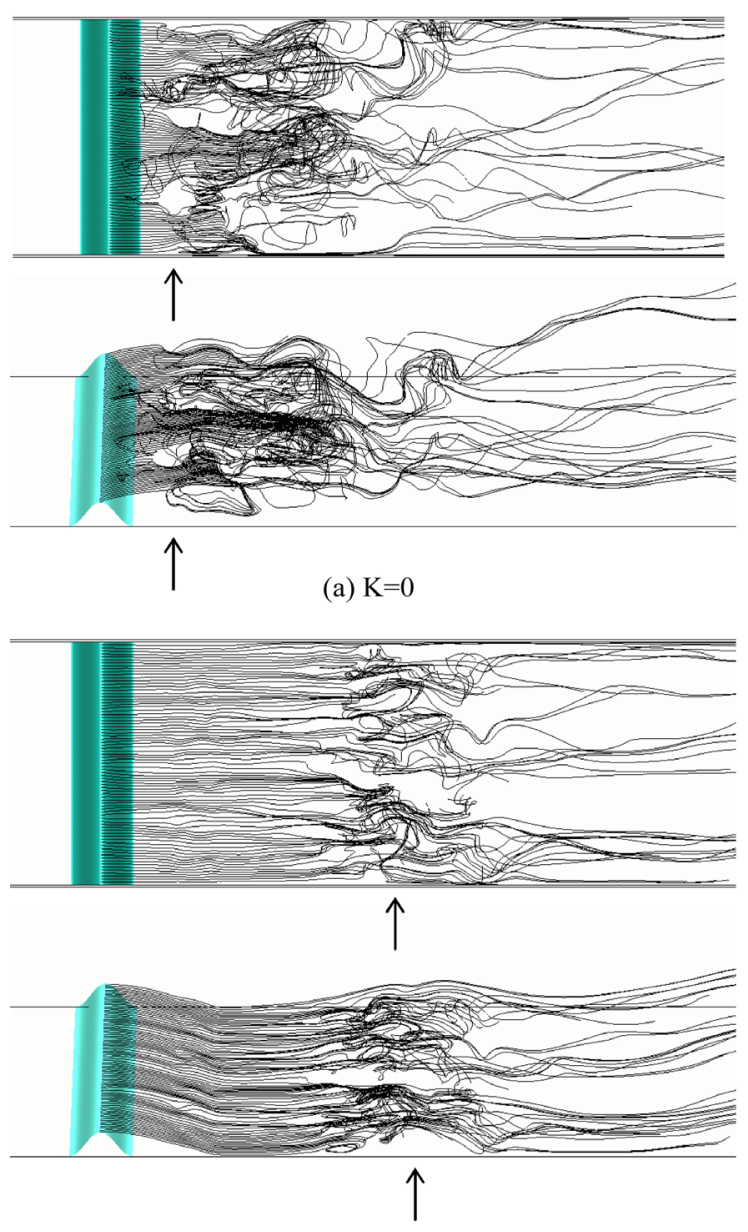

(b) $\mathrm{K}=1.3$

Figure 10. Relationship between the stratification parameter, $K$, and the time-averaged value (non-dimensional time $=100-200)$ of the drag coefficient, $C d, R e=2000$.

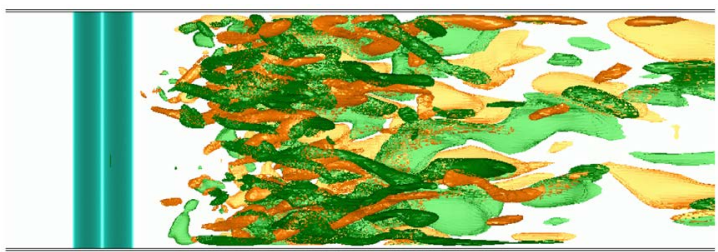

(a) $\mathrm{K}=0$

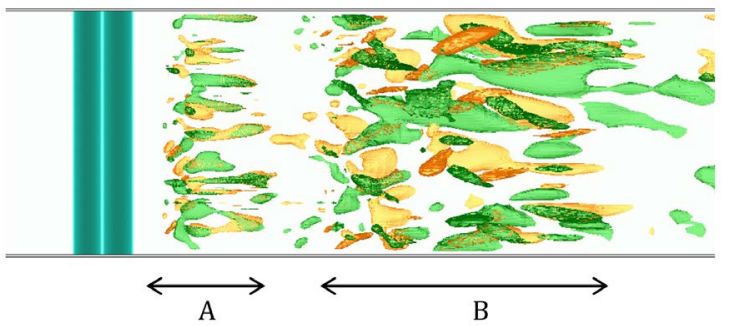

(b) $\mathrm{K}=1.3$

Figure 11. Isosurfaces of the streamwise $(x)$ component of the vorticity, $\omega_{x}$, for the flow in Figure 10. Isosurfaces are drawn for $\omega_{x}= \pm 2, R e=2000$. 


\subsection{Flow over the Hill $(0 \leq K \leq 1.3)$, Time-Averaged Field}

Subsequently, the characteristics of the time-averaged flow field from the 2-D and 3- $D$ simulations are examined. Figure 7 and Figure 8 respectively show the streamlines of released virtual fluid particles and the corresponding spanwise $(y)$ vorticity, $\omega_{y}$, of the time-averaged flows. For both the 2- $D$ and 3-D flows, time-averaging is performed over the non-dimensional time period between $t=$ 100 and $t=200$. For the case of the 3- $D$ flow, spanwise spatial averaging is also performed. Figure 9 illustrates the relationship between the stability and the streamwise $(x)$ length of the separation bubble formed behind the hill, $S R$, that is the streamwise distance from the hill center $(x=0)$ at which the shear layer that separated from the vicinity of the hill top becomes reattached. In Figure 9, $S R$ is normalized by the hill height, $h$.

The trends of the variation of $S R / h$ with respect to the stability, $K$, are similar between the 2- $D$ and 3- $D$ simulations: the value of $S R / h$ increases with increasing stability between $K=0$ and $K=0.8$ while it deceases with increasing stability for $K>0.8$. From the distribution of the vorticity (Figure 8 ), it can be speculated that the following mechanism accounts for the value of $S R / h$ at $K=0.8$ being larger than that at $K=0$, in both the 2-D and 3- $D$ simulations. Due to the effect of the stable stratification, a large velocity deficit occurs in the vicinity of the surface of the hill, and the vorticity (momentum) caused by the hill surface decreases as illustrated by the decreased number of the isolines for vorticity. As a result, the downstream distance at which the separated shear layer becomes reattached increases with increasing stability between $K=0$ and $K=0.8$.

The mechanism responsible for the decreasing values of $S R / h$ with increasing stability for $K>0.8$ is likely identical in the 2-D and 3-D simulations. In this high stability regime, lee waves are excited downstream of the hill unlike at the stabilities of $K=0$ and $K=0.8$. These lee waves induce strong downward flows, which cause the values of $S R / h$ to be significantly smaller than those at $K=0$ and $K=0.8$. Particularly at $K=1.3$, the lee waves are accompanied by strong downward flows immediately downstream of the hill which exceed the uniform inflow velocity, $U$. These strong downward flows reduce the value of $S R / h$ significantly. The difference in the streamwise length of the separation bubble, $S R / h$, between the 2- $D$ and 3- $D$ simulations is evident for both $K=0$ and $K=0.8$ (Figure 9). This result is also visible in the instantaneous fields of the streamlines and the vorticity (Figure 6 and Figure 7). In contrast, at $K=1$, the values of $S R / h$ are similar in the 2-D and 3-D simulations. At $K=1.3$, the values of $S R / h$ become identical for the 2-D and 3-D simulations. This result is attributable to the structure of the spanwise $(y)$ flow structure as discussed in detail in the following subsection.

\subsection{Spanwise (y) Flow Structure in Neutral Conditions $(K=0)$ and Highly Stable Conditions $(K=1.3)$}

The spanwise $(y)$ flow structure is analyzed for neutral $(K=0)$ and highly stable $(K$ 
$=1.3$ ) conditions. Streamlines traced by virtual particles released from the top of the hill and the streamwise $(x)$ component of the vorticity, $\omega_{x}(=\partial w / \partial y-\partial v / \partial z)$, of the 3- $D$ flow are shown in Figure 10 and Figure 11, respectively. At $K=0$, the flow varies in the spanwise $(y)$ direction in a complex manner, suggesting that the flow has become three-dimensional (Figure 10(a)). The three-dimensionality of the complex flow has led to the emergence of longitudinal vortices that entwine with one another over a broad area downstream (Figure 11(a)). Furthermore, variation in the strength of vortex roll-up (separation of streamlines) is evident (arrows in Figure 10(a)) as was also observed in numerically simulated 3- $D$ flows around a cylinder [19] [20].

At $K=1.3$, lee-waves causes strong downward flows immediately downstream of the hill. These downward flows occur almost homogeneously in the $y$-direction. Consequently, the values of $\omega_{x}$ are smaller at $K=1.3$ compared to $K=0$, and the structure of the isosurfaces of $\omega_{x}$ are linear and short in the streamwise $(x)$ direction (area $A$ in Figure 11(b)). In other words, the spanwise ( $y$ ) flow component is strongly inhibited, and the flow becomes approximately two-dimensional in the $x$ and $z$ directions. However, in the vicinity of the rotors induced by the upward flow of the lee-waves further downstream of the hill, the flow becomes heterogeneous in the $y$-direction (indicated by arrows in Figure $10(\mathrm{~b})$ and area $B$ in Figure 11(b)). Therefore, strong disturbances are locally generated within and near the rotors even in highly stably stratified flows.

\section{Summary}

Three-dimensional direct numerical simulations (DNS) were conducted for flows over a hill in neutral and stably stratified conditions. The present study followed onto our previous two-dimensional DNS studies on flows over a hill in stably stratified conditions and provided a more realistic simulation of atmospheric flows than our previous studies. In the 3- $D$ simulation, a hill with a constant cross-section in the spanwise $(y)$ direction was placed in the computational domain. The spanwise dimension of the computational domain was eight times the height of the hill. As in our previous research [5], no-slip conditions were imposed only on the hill surface and the surface downstream of the hill; on the surface upstream of the hill, slip conditions were imposed. This set of surface conditions was adopted to investigate the flow over the hill without considering the effect of the ground boundary layer that would develop upstream of the hill. The analyses of the results focused on the effect of stable stratification on the non-periodic separation and reattachment of the flow behind the hill. The simulated 3- $D$ flows were also discussed in terms of the differences and similarities with the simulated 2- $D$ flows.

In neutral $(K=0)$ and weakly stable $(K=0.8)$ conditions, the characteristics of the simulated 3- $D$ flow over the hill were significantly different from those of the simulated 2- $D$ flow over the hill. In both the 2- $D$ and 3- $D$ flows, vortices were periodically shed from the hill. However, unlike in the 2- $D$ flow, the shear layers 
separated from the hill top area in the 3- $D$ flow did not become reattached on the downstream side of the hill and curled upward at some distance downstream of the hill. Subsequently, vortices formed that were not connected to the surface downstream of the hill. These vortices were then shed and advected downstream. As a result, the shear layer which separated from the hill top area became reattached significantly farther downstream (significantly larger value of $S R / h$ ) in the 3- $D$ flow than in the 2- $D$ flow. In both the 2- $D$ and 3- $D$ flows, the normalized downwind distance from the hill top at which the separated shear layer is reattached, $S R / h$, was significantly larger at $K=0.8$ than at $K=0$. The increased value of $S R / h$ at $K=0.8$ was attributable to the reduction in vorticity (momentum) caused by the surface of the hill.

In highly stable conditions $(K=1,1.3)$, the characteristics of the 3- $D$ flow in the vicinity of the hill were remarkably similar to those of the 2- $D$ flow. The degree of the similarities was higher at $K=1.3$. This result is attributable to the spanwise $(y)$ flow structure, that is, immediately downstream of the hill, strong downward flow was induced homogeneously in the $y$-direction by lee waves. Furthermore, the streamwise $(x)$ component of the vorticity, $\omega_{x}$, was significantly smaller at $K=1$ and $K=1.3$ than at $K=0$, and the isosurfaces of the vorticity component $\omega_{x}$ were linear and short in the streamwise $(x)$ direction. In other words, the spanwise $(y)$ component of the flow was strongly suppressed, and the flow became two dimensional in the $x$ and $z$ directions. Thus, the qualitative behaviors of the flows in the vicinity of the hill were similar between the simulated 2- $D$ and 3- $D$ flows in highly stable conditions. In addition, with the influence of the downward flow of the lee waves, the values of $S R / h$ became shorter in highly stable conditions than in neutral $(K=0)$ and weakly stable $(K=0.8)$ conditions in both the 2-D and 3- $D$ simulations. Finally, in highly stable conditions, in the region further downstream of the hill at which rotors were induced by the upward flow of the lee waves, strong disturbances were generated locally.

\section{References}

[1] Paisley, M.F., Castro, I.P. and Rockliff, N.J. (1994) Steady and Unsteady Computations of Strongly Stratified Flows over a Vertical Barrier. In: Stably Stratified Flows: Flow and Dispersion over Topography, Clarendon Oxford University Press, Oxford, 39-59.

[2] Snyder, W.H. (1985) Fluid Modeling of Pollutant Transport and Diffusion in Stably Stratified Flows over Complex Terrain. Annual Review of Fluid Mechanics, 17, 239-266. https://doi.org/10.1146/annurev.fl.17.010185.001323

[3] Uchida, T. and Ohya, Y. (1997) A Numerical Study of Stably Stratified Flows over a Two-Dimensional Hill-Part. I Free-Slip Condition on the Ground. Journal of Wind Engineering \& Industrial Aerodynamics, 67 \& 68, 493-506.

[4] Uchida, T. and Ohya, Y. (1998) Unsteady Characteristics of Stably Stratified Flows past a Two-Dimensional Hill in a Channel of Finite Depth. Journal of Japan Society of Fluid Mechanics, 17, 45-56.

[5] Uchida, T. and Ohya, Y. (1999) Unsteady Characteristics of Stably Stratified Flows past a Two-Dimensional Hill in a Channel of Finite Depth-Part II. Effect of Stable 
Stratification on the Separated-Reattached Flow. Journal of Japan Society of Fluid Mechanics, 18, 308-320.

[6] Uchida, T. and Ohya, Y. (1998) Large Eddy Simulation of Stably Stratified Flows past a Two-Dimensional Hill in a Channel of Finite Depth. Journal of Applied Me chanics, JSCE, 1, 615-624.

[7] Uchida, T. and Ohya, Y. (1999) Numerical Study of Topography-Induced Local Severe Winds in Stably Stratified Fluid. Journal of Applied Mechanics, JSCE, 2, 583-592.

[8] Uchida, T. and Ohya, Y. (2000) Two-Dimensional Numerical Simulation of Stably-Stratified Flows past a Cosine-Shaped Hill-Effect of the Grid Resolution and the Numerical Method. Journal of Applied Mechanics, JSCE, 3, 729-736.

[9] Uchida, T. and Ohya, Y. (2001) Numerical Investigation of Local Severe Winds Induced by Lee-Wave Breaking over Topography in Stably Stratified Flows. Journal of Applied Mechanics, JSCE, 4, 655-664.

[10] Uchida, T. and Ohya, Y. (2001) Numerical Study of Stably Stratified Flows over a Two-Dimensional Hill in a Channel of Finite Depth. Fluid Dynamics Research, 29, 227-250. https://doi.org/10.1016/S0169-5983(01)00025-9

[11] Ohya, Y., Uchida, T. and Nagai, T. (2013) Near Wake of a Horizontal Circular Cylinder in Stably Stratified Flows. Open Journal of Fluid Dynamics, 3, 311-320. https://doi.org/10.4236/ojfd.2013.34038

[12] Lilly, D.K. (1978) A Severe Downslope Windstorm and Aircraft Turbulence Induced by a Mountain Wave. Journal of the Atmospheric Sciences, 35, 59-77. https://doi.org/10.1175/1520-0469(1978)035<0059:ASDWAA >2.0.CO;2

[13] Saito, K. (1998) Wind Engineers. JAWE, 75, 79-84.

[14] Katsura, J. (1999) Wind Engineers. JAWE, 78, 63-66.

[15] Kim, J. and Moin, P. (1985) Application of a Fractional-Step Method to Incompressible Navier-Stokes Equations. Journal of Computational Physics, 59, 308-323. https://doi.org/10.1016/0021-9991(85)90148-2

[16] Kawamura, T., et al. (1986) Computation of High Reynolds Number Flow around A Circular Cylinder with Surface Roughness. Fluid Dynamics Research, 1, 145-162. https://doi.org/10.1016/0169-5983(86)90014-6

[17] Kawamura, T., et al. (1997) High-Order Finite-Difference Method for Incompressible Flows using Collocated Grid System. Transactions of the Japan Society of Mechanical Engineers, B 63, 3247-3254. https://doi.org/10.1299/kikaib.63.3247

[18] Uchida, T. and Ohya, Y. (2002) Numerical Investigation of an Unsteady Vortex Shedding from a Two-Dimensional Steep Ridge. Journal of Applied Mechanics, JSCE, 5, 735-742.

[19] Izumi, H., et al. (1994) Three-Dimensional Flow Analysis around a Circular Cylinder: 1st Report, In the Case of a Stationary Circular Cylinder. Transactions of the Japan Society of Mechanical Engineers, B 63, 3797-3804. https://doi.org/10.1299/kikaib.60.3797

[20] Suzuki, M. and Kuwahara, K. (1992) Stratified Flow past a Bell-Shaped Hill. Fluid Dynamics Research, 9, 1-18. https://doi.org/10.1016/0169-5983(92)90055-2 